This item was submitted to Loughborough's Research Repository by the author.

Items in Figshare are protected by copyright, with all rights reserved, unless otherwise indicated.

\title{
Impulsive gravitational waves generated by null particles in de Sitter and anti-de Sitter backgrounds
}

PLEASE CITE THE PUBLISHED VERSION

PUBLISHER

(C) American Physical Society

LICENCE

CC BY-NC-ND 4.0

REPOSITORY RECORD

Podolsky, J., and J.B. Griffiths. 2019. "Impulsive Gravitational Waves Generated by Null Particles in De Sitter and Anti-de Sitter Backgrounds". figshare. https://hdl.handle.net/2134/1723. 


\title{
Impulsive gravitational waves generated by null particles in de Sitter and anti-de Sitter backgrounds
}

\author{
Jiří Podolský \\ Department of Theoretical Physics, Faculty of Mathematics and Physics, Charles University, V Holešovičkách 2, 18000 Prague 8, \\ Czech Republic \\ Jerry B. Griffiths \\ Department of Mathematical Sciences, Loughborough University, Loughborough, Leicestershire LE11 3TU, United Kingdom
}

(Received 7 May 1997)

\begin{abstract}
By boosting the metric of the Schwarzschild-de Sitter and Schwarzschild-anti-de Sitter space-times in the ultrarelativistic limit as the mass parameter vanishes, impulsive gravitational waves are obtained in backgrounds with a nonzero cosmological constant. We determine the geometry of these impulsive wave surfaces using various coordinate charts. In the de Sitter background they are spheres containing two null particles located at the poles. In the anti-de Sitter background they are hyperboloidal surfaces of constant negative curvature containing a single null particle on the axis of symmetry. The global structure and conformal diagrams for the complete manifolds are also determined. [S0556-2821(97)04620-1]
\end{abstract}

PACS number(s): 04.20.Jb, 04.30.Nk

\section{INTRODUCTION}

In a now classic paper, Aichelburg and Sexl [1] obtained an exact solution of Einstein's equations for a null particle in a Minkowski background by boosting the Schwarzschild metric to the ultrarelativistic limit as the mass becomes arbitrarily small. The resulting space-time is an impulsive $p p$ wave. Generalizations of this solution have been obtained by a number of authors [2-5] by similarly boosting the Kerr or Kerr-Newman solutions.

A similar approach has been adopted by Hotta and Tanaka [6] in boosting the Schwarzschild-de Sitter metric to a similar limit. Effectively, they consider this as a perturbation of the de Sitter metric and boost the particle to the ultrarelativistic limit as its mass vanishes in an appropriate way. This approach also results in impulsive gravitational waves. However, the background in this case is not flat and the wavefronts are not simple planes in Minkowski space. Rather they are surfaces of constant curvature in the de Sitter background. The calculations can easily be adapted to describe impulsive gravitational waves in an anti-de Sitter background. However, the global properties of the solution in this case differ significantly.

It is the purpose of the present paper to clarify certain aspects of these solutions and to investigate the geometrical properties of the impulsive wave surfaces as they would be seen by different families of observers.

\section{BOOSTING THE SCHWARZSCHILD-de SITTER SOLUTION}

Let us start with the Schwarzschild-de Sitter solution in the familiar form

$$
\begin{aligned}
d s^{2}= & \left(1-\frac{r^{2}}{a^{2}}-\frac{2 m}{r}\right) d t^{2}-\left(1-\frac{r^{2}}{a^{2}}-\frac{2 m}{r}\right)^{-1} d r^{2} \\
& -r^{2}\left(d \theta^{2}+\sin ^{2} \theta d \phi^{2}\right),
\end{aligned}
$$

in which the cosmological constant is given by $\Lambda=3 / a^{2}$ and $m$ is the mass parameter. In the limit when $m=0$, this is simply the de Sitter solution which, as is well known, can be represented as a four-dimensional hyperboloid

$$
Z_{0}^{2}-Z_{1}^{2}-Z_{2}^{2}-Z_{3}^{2}-Z_{4}^{2}=-a^{2}
$$

embedded in a five-dimensional Minkowski space-time

$$
d s^{2}=d Z_{0}^{2}-d Z_{1}^{2}-d Z_{2}^{2}-d Z_{3}^{2}-d Z_{4}^{2}
$$

For $m=0$, this can be achieved by putting

$$
\begin{gathered}
Z_{0}=\sqrt{a^{2}-r^{2}} \sinh (t / a), \\
Z_{4}= \pm \sqrt{a^{2}-r^{2}} \cosh (t / a), \\
Z_{1}=r \cos \theta \\
Z_{2}=r \sin \theta \cos \phi \\
Z_{3}=r \sin \theta \sin \phi .
\end{gathered}
$$

From this it can immediately be observed that the origin $r=0$ corresponds to the two world lines

$$
\left(Z_{0}, Z_{1}, Z_{2}, Z_{3}, Z_{4}\right)=(a \sinh (t / a), 0,0,0, \pm a \cosh (t / a))
$$

along the hyperboloid, as indicated in Fig. 1 in which the coordinates $Z_{2}$ and $Z_{3}$ are suppressed. It may seem surprising that the Schwarzschild-de Sitter solution (2.1) does not just have a single source in the limit when the mass is small. However, it is known that the coordinates of Eq. (2.1) do not cover the complete space-time whose analytic extension must include both the black hole and white hole parts. This fact can be deduced from its conformal structure (see, e.g., Bičák and Podolský [7]). 


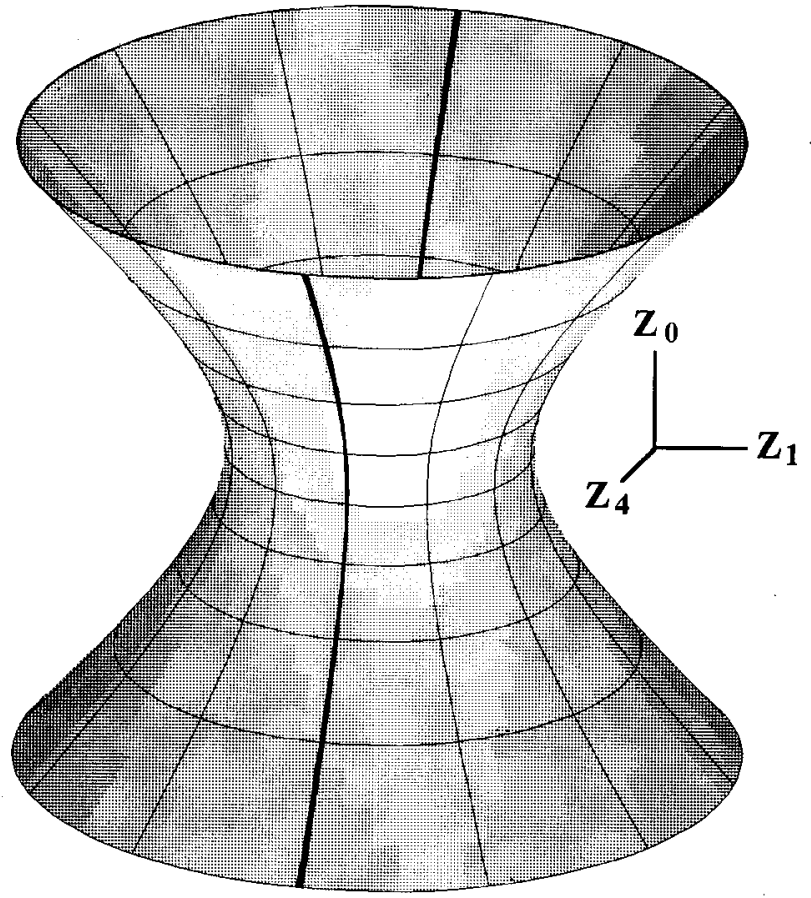

FIG. 1. The de Sitter space-time represented as a fourdimensional hyperboloid embedded in a five-dimensional Minkowski space-time. The coordinates $Z_{2}$ and $Z_{3}$ have been suppressed, and the timelike world lines are indicated by the two test particles which correspond to the weak limit of the source of the Schwarzschild-de Sitter space-time.

Following the approach of Aichelburg and Sexl [1], we may now boost the singularity representing the point mass in the Schwarzschild-de Sitter solution to the limit in which its speed approaches that of light and its mass is scaled to zero in an appropriate way.

As observed by Hotta and Tanaka [6], in this case it is first appropriate to take the linear approximation of Eq. (2.1) for small $m$ in the form

$$
\begin{aligned}
d s^{2}= & \left(1-\frac{r^{2}}{a^{2}}-\frac{2 m}{r}\right) d t^{2}-\left(1-\frac{r^{2}}{a^{2}}\right)^{-1} \\
& \times\left[1+\left(1-\frac{r^{2}}{a^{2}}\right)^{-1} \frac{2 m}{r}\right] d r^{2}-r^{2}\left(d \theta^{2}+\sin ^{2} \theta d \phi^{2}\right) .
\end{aligned}
$$

This can be considered as a first order perturbation of the de Sitter line element. Using the coordinates $Z_{i}$ of Eqs. (2.4), the first order perturbation of the line element (2.3) can be written as

$$
\begin{aligned}
d s_{1}^{2}= & -\frac{2 m a^{2}}{r}\left[\left(\frac{Z_{4} d Z_{0}-Z_{0} d Z_{4}}{Z_{4}^{2}-Z_{0}^{2}}\right)^{2}\right. \\
& \left.+\frac{a^{2}}{r^{2}}\left(\frac{Z_{0} d Z_{0}-Z_{4} d Z_{4}}{Z_{4}^{2}-Z_{0}^{2}}\right)^{2}\right]
\end{aligned}
$$

where $r=\left(a^{2}+Z_{0}^{2}-Z_{4}^{2}\right)^{1 / 2}$. A boost can then be performed in any direction orthogonal to $Z_{4}$ (a boost in the $Z_{4}$ direction simply corresponds to a time shift). Taking the boost in the $Z_{1}$ direction, we can put

$$
\begin{gathered}
Z_{0} \rightarrow \frac{Z_{0}+v Z_{1}}{\sqrt{1-v^{2}}}, \quad Z_{1} \rightarrow \frac{v Z_{0}+Z_{1}}{\sqrt{1-v^{2}}}, \\
Z_{2} \rightarrow Z_{2}, \quad Z_{3} \rightarrow Z_{3}, \quad Z_{4} \rightarrow Z_{4},
\end{gathered}
$$

where $v$ is the boost parameter. It can then be seen that, in the limit as $v \rightarrow 1$, the linear perturbation term vanishes everywhere except on the hypersurface $Z_{0}+Z_{1}=0$. Then, by also scaling the mass according to

$$
m=p \sqrt{1-v^{2}}
$$

where $p$ is a constant, it can be seen that the only nonvanishing term in Eq. (2.7) is that which becomes a multiple of $\left(d Z_{0}+d Z_{1}\right)^{2}$. The line element thus takes the form

$$
\begin{aligned}
d s^{2}= & d Z_{0}^{2}-d Z_{1}^{2}-d Z_{2}^{2}-d Z_{3}^{2}-d Z_{4}^{2} \\
& -2 p a^{2} H\left(Z_{4}\right) \delta\left(Z_{0}+Z_{1}\right)\left(d Z_{0}+d Z_{1}\right)^{2},
\end{aligned}
$$

where, putting

$$
\begin{gathered}
x^{2}=\frac{\left(Z_{0}+v Z_{1}\right)^{2}}{1-v^{2}}, \\
H\left(Z_{4}\right) \delta\left(Z_{0}+Z_{1}\right)=\lim _{v \rightarrow 1} \frac{1}{\sqrt{1-v^{2}}} \frac{\left(a^{2}-Z_{4}^{2}\right) Z_{4}^{2}+\left(a^{2}+Z_{4}^{2}\right) x^{2}}{\left(a^{2}-Z_{4}^{2}+x^{2}\right)^{3 / 2}\left(Z_{4}^{2}-x^{2}\right)^{2}} .
\end{gathered}
$$

In evaluating this limit, we follow Hotta and Tanaka [6], in making use of the identity

$$
\lim _{v \rightarrow 1} \frac{1}{\sqrt{1-v^{2}}} f\left(\frac{\left(Z_{0}+v Z_{1}\right)^{2}}{1-v^{2}}\right)=\delta\left(Z_{0}+Z_{1}\right) \int_{-\infty}^{\infty} f\left(x^{2}\right) d x
$$

which can easily be proven in the theory of distributions. However, in this case, the integrand involved contains singularities at $x= \pm Z_{4}$. Nevertheless, it is possible to remove the effect of these by first making a singular coordinate transformation such that

$$
\begin{aligned}
d Z_{0}-d Z_{1} \rightarrow & d Z_{0}-d Z_{1}-\lim _{v \rightarrow 1} \frac{4 p a^{3}}{\sqrt{1-v^{2}}} \\
& \times\left(a^{2}-\frac{\left(Z_{0}+Z_{1}\right)^{2}}{1-v^{2}}\right)^{-2}\left(d Z_{0}+d Z_{1}\right) .
\end{aligned}
$$

Then, using Eq. (2.11), it can be shown that the function $H\left(Z_{4}\right)$ in the line element (2.9) takes the form

$$
H\left(Z_{4}\right)=-\frac{2}{a^{2}}\left[2-\frac{Z_{4}}{a} \ln \left|\frac{a+Z_{4}}{a-Z_{4}}\right|\right] .
$$

It can thus be seen that the resulting space-time differs from the de Sitter space-time only by the inclusion of an impulsive wave located on the null hypersurface given by 


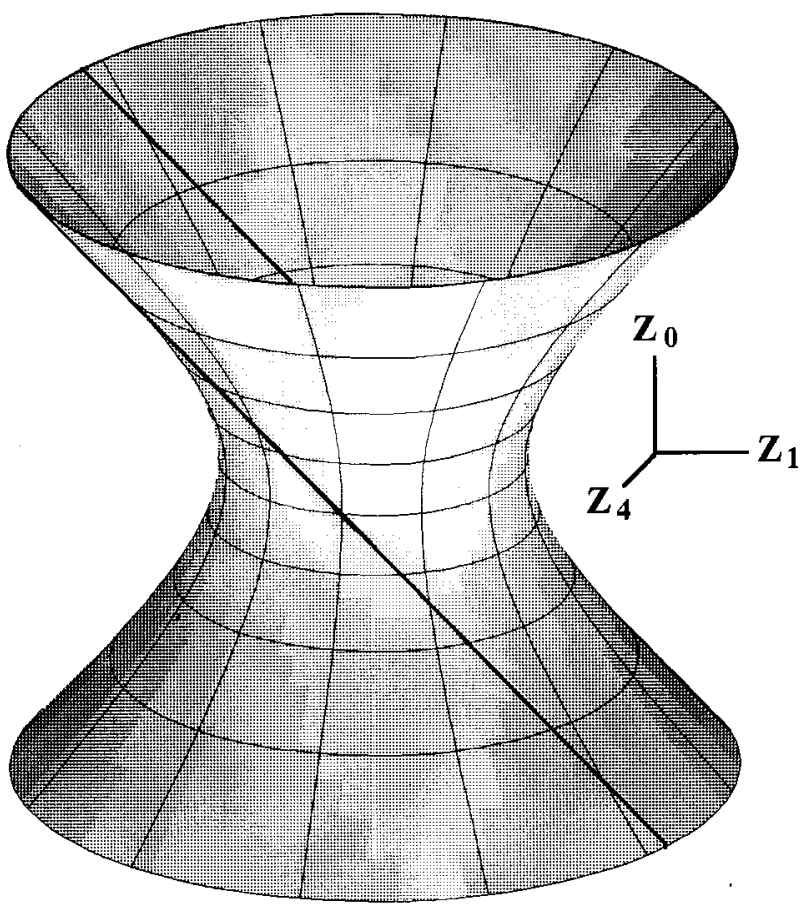

FIG. 2. The de Sitter space-time represented as a fourdimensional hyperboloid embedded in a five-dimensional Minkowski space-time with the coordinates $Z_{2}$ and $Z_{3}$ suppressed. The null world lines are indicated by the two particles which correspond to the source of the Schwarzschild-de Sitter space-time boosted to the limit in which its velocity is that of light and its mass vanishes.

$$
Z_{0}+Z_{1}=0, \quad Z_{2}^{2}+Z_{3}^{2}+Z_{4}^{2}=a^{2} .
$$

It may be observed that the shock wave is a two-sphere in the five-dimensional Minkowski space at any time $Z_{0}$.

In addition, as shown in [6], the singularities that were originally located at $r=0$ have now been boosted to become two null particles described by $\delta$ functions in the energymomentum tensor on the wave surface. These have the world lines

$$
Z_{0}+Z_{1}=0, \quad Z_{2}=Z_{3}=0, \quad Z_{4}= \pm a .
$$

These are the two null straight lines on the four-dimensional hyperboloid as indicated in Fig. 2.

\section{GLOBAL COORDINATES $(\Lambda>0)$}

Let us now investigate the geometry of the shock wave in the de Sitter background. To do this, we set up some appropriate coordinate system. At each event, we introduce some "time" and "space" labels. We can then ask the question, what are the space coordinates of the shock wave at a given "synchronized" time? Of course, although the geometry is unique, the coordinates can be chosen arbitrarily, and so that the coordinate shape is not unique. In practice, however, "comoving coordinates" can always be introduced, and so the coordinate shape of the shock wave will correspond to its shape as seen by the associated family of observers.

In fact there are a number of privileged families of observers in a de Sitter space. These correspond to those in which the spatial sections at any synchronous time have constant curvature. We will consider the three cases of positive, zero, and negative curvature. Of these the most natural choices of coordinates are those associated with spatial sections of constant positive curvature. These will be considered in the present section, with the other cases considered in Sec. IV. Further properties of the coordinates used for the de Sitter space-time and the relations between them have been described by Eriksen and Grøn [8], although in a slightly different notation.

The most natural set of coordinates covering the fourdimensional hyperboloid (2.2) is the set of "global coordinates" given by

$$
\begin{gathered}
Z_{0}=a \sinh (\tau / a), \\
Z_{1}=a \cosh (\tau / a) \cos \chi, \\
Z_{4}=a \cosh (\tau / a) \sin \chi \cos \theta, \\
Z_{2}=a \cosh (\tau / a) \sin \chi \sin \theta \cos \phi, \\
Z_{3}=a \cosh (\tau / a) \sin \chi \sin \theta \sin \phi,
\end{gathered}
$$

in which the coordinates $\theta$ and $\phi$ are different from those of Sec. II. Indeed the same symbols are used for a number of different coordinate parametrizations below. With this, the line element becomes

$d s^{2}=d \tau^{2}-a^{2} \cosh ^{2}(\tau / a)\left[d \chi^{2}+\sin ^{2} \chi\left(d \theta^{2}+\sin ^{2} \theta d \phi^{2}\right)\right]$.

In this case, the privileged family of timelike observers have world lines on which $\chi, \theta$, and $\phi$ are constant. For this family, the three-spaces $\tau=$ const, which represent the universe at any time, have the geometry of three-spheres $S^{3}$ which appear to contract to a minimum size at $\tau=0$, and then reexpand.

Let us now return to consider the shock wave, which is given by Eqs. (2.13) with the null particles having the world lines (2.14). Using Eqs. (3.1), we see that the shock wave is located at $\cos \chi=-\tanh (\tau / a)$. Thus, the hyperspherical coordinate $\chi$ monotonically increases from $\chi=0$ as $\tau \rightarrow-\infty$ to $\chi=\pi / 2$ at $\tau=0$ and $\chi=\pi$ as $\tau \rightarrow+\infty$. This has a natural physical interpretation shown in Fig. 3. As the synchronous (proper) time $\tau$ of comoving observers at fixed points $P$ given by constant $\chi_{0}, \theta_{0}$, and $\phi_{0}$ increases, the impulse propagates from the "north pole" $(\chi=0)$ to the "south pole" $(\chi=\pi)$ of the closed space $S^{3}$. Moreover, the radius of $S^{3}$ is given by $R=a \cosh (\tau / a)$; i.e., as $\tau$ increases, for $\tau<0$, the space contracts to a minimum radius $a($ at $\tau=0$ ) and then reexpands for $\tau>0$.

This is visualized in Fig. 3 where the coordinate $\phi$ was suppressed so that the evolution of the universe is represented by a sequence of two-spheres (with coordinates $\chi$ and $\theta$ ) of which only hemispheres are shown. At any time, the impulse is here given by a (semi)circle which is located exactly in the circular intersection of the corresponding sphere with a cylinder of radius $a$. Indeed, the distance of the impulse from the axis through the poles is given by $R \sin \chi=a \cosh (\tau / a) \sqrt{1-\tanh ^{2}(\tau / a)} \equiv a$. The null particles 


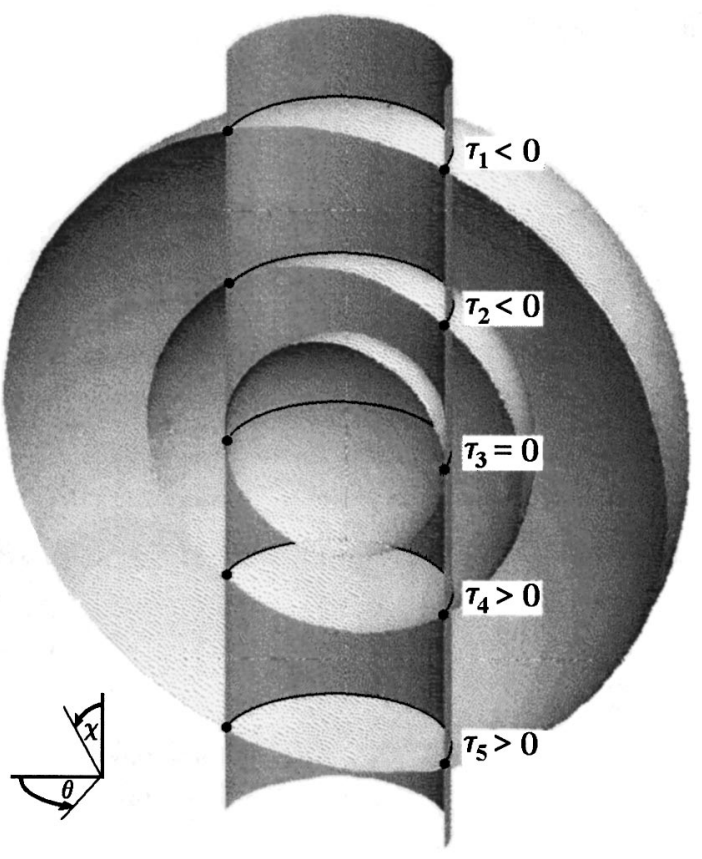

FIG. 3. In natural coordinates the de Sitter space-time is a closed universe with the spatial geometry of a three-sphere whose radius is given by $R=a \cosh (\tau / a)$. Suppressing the coordinate $\phi$, its evolution can be visualized as a sequence of two-spheres which are indicated here by hemispheres which contract to a minimum size and then reexpand. At any time, the impulsive wave is a two-sphere represented here by a semicircle located at the circular intersection of a cylinder of radius $a$ with the corresponding sphere representing the universe. This propagates from the "north pole" $\chi=0$ as $\tau \rightarrow-\infty$ across the equator $\chi=\pi / 2$ at $\tau=0$ to the "south pole", $\chi=\pi$ as $\tau \rightarrow \infty$ while the whole universe contracts to a minimum size $R=a$ at $\tau=0$ and then reexpands. which are the singular sources of the wave are located at $\theta=0$ and $\theta=\pi$, as follows immediately from Eqs. (2.14) and (3.1).

In order to investigate the global conformal structure of the space-time, it is useful to introduce the conformal time $\eta_{p}$ defined by

$$
\eta_{p}=2 \arctan \left(e^{\tau / a}\right)
$$

Then $\sinh (\tau / a)=-\cot \eta_{p}, \cosh (\tau / a)=1 / \sin \eta_{p}$, and the line element (3.2) becomes

$$
d s^{2}=\frac{a^{2}}{\sin ^{2} \eta_{p}}\left[d \eta_{p}^{2}-d \chi^{2}-\sin ^{2} \chi\left(d \theta^{2}+\sin ^{2} \theta d \phi^{2}\right)\right] .
$$

In this case, the appropriate conformal diagram which is shown in Fig. 4 covers the coordinate ranges from $\eta_{p}=0$ $(\tau=-\infty)$ to $\eta_{p}=\pi(\tau=\infty)$, and from $\chi=0$ to $\chi=\pi$.

It may then be observed that the condition $Z_{0}+Z_{1}=0$ corresponds to the single root $\chi=\eta_{p}$, and hence that

$$
\delta\left(Z_{0}+Z_{1}\right)=\frac{1}{a} \delta\left(\chi-\eta_{p}\right)
$$

The impulsive wave in the conformal diagram in Fig. 4 is simply located along the line $\chi=\eta_{p}$. Moreover, since the function $H\left(Z_{4}\right)$ only need be evaluated for $\chi=\eta_{p}$, it can be seen that the impulsive addition to the de Sitter line element (3.4) is given by

$$
d s_{1}^{2}=4 p a\left[2-\cos \theta \ln \left|\frac{1+\cos \theta}{1-\cos \theta}\right|\right] \delta\left(\eta_{p}-\chi\right)\left(d \eta_{p}-d \chi\right)^{2} .
$$

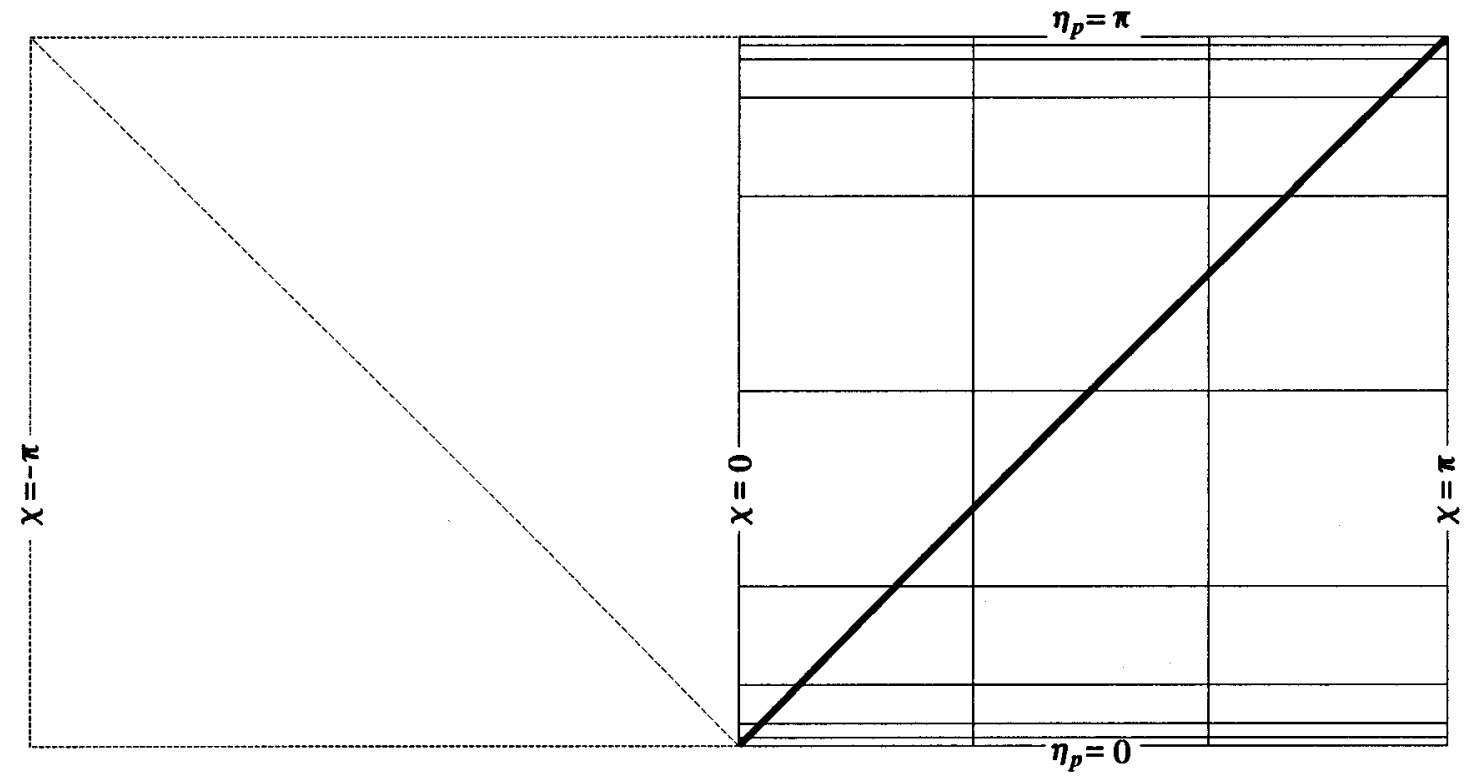

FIG. 4. The conformal diagram of the de Sitter space-time with natural global coordinates $\eta_{p}, \chi$. Each point of the diagram represents a two-sphere parametrized by coordinates $\theta, \phi$. The lines $\chi=0$ and $\chi=\pi$ are identified. In contrast to Minkowski space-time, de Sitter has a spacelike future and past infinity for null and timelike lines. The impulsive wave is located along $\chi=\eta_{p}$ with two singular points sources at $\theta=0$ and $\theta=\pi$. 
From this, it can immediately be seen that the two null particles are located at the points $\theta=0$ and $\theta=\pi$, which correspond to the opposite poles of the spherical wave surface.

\section{OTHER COORDINATE CHARTS $(\Lambda>0)$}

Although the coordinates introduced in the previous section are the most natural in the sense that they cover the entire de Sitter space-time and provide a simple interpretation of the shock geometry, it is also appropriate to consider some alternative coordinate systems as they can be interpreted in terms of different families of observers.

Taking the case in which spatial sections at synchronous times have zero curvature, we can introduce the conformally flat coordinates

$$
\begin{gathered}
Z_{0}=\frac{1}{2 \eta}\left[a^{2}-\eta^{2}+(x-a)^{2}+y^{2}+z^{2}\right], \\
Z_{1}=\frac{a}{\eta}(x-a), \\
Z_{2}=\frac{a}{\eta} y, \\
Z_{3}=\frac{a}{\eta} z, \\
Z_{4}=\frac{1}{2 \eta}\left[a^{2}+\eta^{2}-(x-a)^{2}-y^{2}-z^{2}\right] .
\end{gathered}
$$

With this, the line element becomes

$$
d s^{2}=\frac{a^{2}}{\eta^{2}}\left(d \eta^{2}-d x^{2}-d y^{2}-d z^{2}\right) .
$$

When coming to describe the geometry of the impulsive wave in these coordinates, it is convenient to put

$x=\rho \cos \theta, \quad y=\rho \sin \theta \cos \phi, \quad z=\rho \sin \theta \sin \phi$,

where $\rho \in[0, \infty), \theta \in[0, \pi], \phi \in[0,2 \pi)$, so that

$$
\rho^{2}=x^{2}+y^{2}+z^{2},
$$

and then we have

$$
Z_{0}+Z_{1}=-\frac{1}{2 \eta}\left(\eta^{2}-\rho^{2}\right)
$$

and

$$
Z_{4}=\frac{1}{2 \eta}\left(\eta^{2}-\rho^{2}+2 a x\right) .
$$

Thus, on the impulsive wave on which $Z_{0}+Z_{1}=0$, we have $\eta= \pm \rho$ and

$$
Z_{4}=\frac{a x}{\eta}=a \frac{\rho}{\eta} \cos \theta
$$

It may be noted that, in order to include the maximum analytic extension of the space-time, it is necessary to permit $\eta$ to cover the range $(-\infty, \infty)$. In addition, from Eq. (4.3) it follows that

$$
\delta\left(Z_{0}+Z_{1}\right)=\delta(\eta-\rho)+\delta(\eta+\rho),
$$

which indicates that the impulsive wave has two components,

$$
\begin{gathered}
\text { when } \eta=\rho, \quad Z_{4}=a \cos \theta, \\
\text { when } \eta=-\rho, \quad Z_{4}=-a \cos \theta \text {. }
\end{gathered}
$$

Combining these, we see that the impulsive addition to the de Sitter line element (4.2) is given by

$$
\begin{aligned}
d s_{1}^{2}= & 4 p\left[2-\cos \theta \ln \left|\frac{1+\cos \theta}{1-\cos \theta}\right|\right] \\
& \times\left[\delta(\eta-\rho)(d \eta-d \rho)^{2}+\delta(\eta+\rho)(d \eta+d \rho)^{2}\right] .
\end{aligned}
$$

This looks like two impulses. However, both components are required for the conformal picture to be geodesically complete: Since $\rho \geqslant 0$, the term $\delta(\eta-\rho)$ is nonvanishing only for $\eta \geqslant 0$ while $\delta(\eta+\rho)$ applies only for $\eta \leqslant 0$. Again it can be seen that the two null source particles are located at the poles $\theta=0$ and $\theta=\pi$ on the spherical wave surface.

The conformal diagram of the section $y=z=0$ is shown in Fig. 5. In this, the whole de Sitter universe is covered by two regions in which $\eta \geqslant 0$ and $\eta \leqslant 0$. It is also convenient to use $x=\rho \cos \theta$ where $\theta=0$ or $\pi$. The diagram thus illustrates the paths of the two null particles propagating in opposite directions. The impulsive waves $\rho=\eta$ and $\rho=-\eta$ can also be seen to represent either a contracting two-sphere in an exponentially expanding universe or an expanding twosphere in a contracting universe.

This point can also be clarified by introducing the proper time $\tau$ defined by

$$
\eta= \pm a e^{-\tau / a}
$$

so that the line element (4.2) of de Sitter space-time becomes

$$
d s^{2}=d \tau^{2}-e^{2 \tau / a}\left[d \rho^{2}+\rho^{2}\left(d \theta^{2}+\sin ^{2} \theta d \phi^{2}\right)\right] .
$$

It can thus be seen that the region in which $\eta>0$ corresponds to an exponentially expanding "inflationary" universe. The region included in the conformal diagram in which $\eta<0$ corresponds to a time-reversed exponentially contracting universe.

It may also be observed that the "radius" of the spherical impulsive wave is given by

$$
\rho=|\eta|=a e^{-\tau / a} .
$$

Thus, in the expanding universe with the three-dimensional spaces $\tau=$ const being flat, the impulsive wave is an exponentially contracting two-sphere. Conversely (changing $\tau \rightarrow-\tau)$, it can also be considered as an expanding spherical wave in an exponentially contracting universe. 


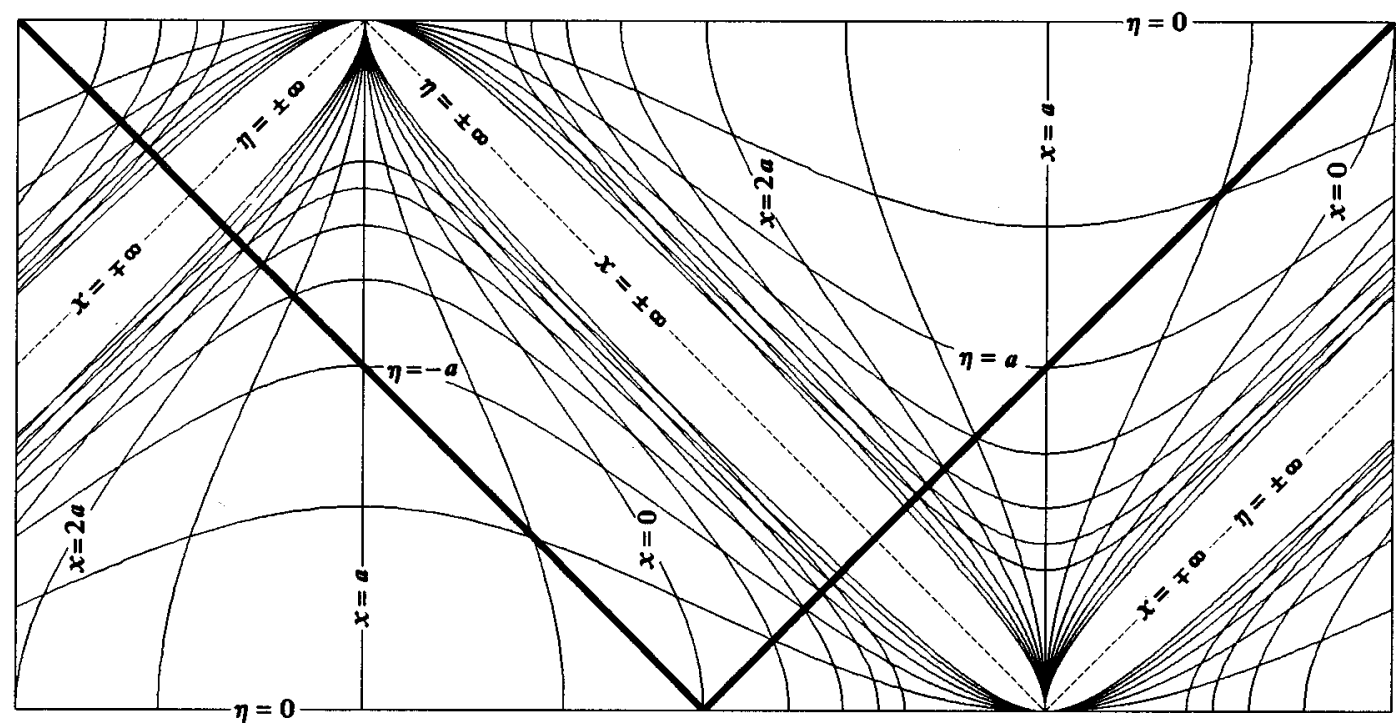

FIG. 5. The conformal diagram of the section $y=z=0$ of the de Sitter space-time with coordinates $\eta, \rho=|x|$. The wave is located along $\eta= \pm \rho$ and represents either an exponentially contracting two-sphere in an exponentially expanding flat inflationary universe or an expanding two-sphere in a contracting space.

Let us finally consider how the impulsive wave would appear to the family of observers which, at any synchronous time, are located in a space of a negative curvature. In this case, the four-dimensional hyperboloid (2.2) can be parametrized by

$$
\begin{gathered}
Z_{0}=a \sinh (\tau / a) \cosh \psi, \\
Z_{1}=a \cosh (\tau / a), \\
Z_{4}=a \sinh (\tau / a) \sinh \psi \cos \theta, \\
Z_{2}=a \sinh (\tau / a) \sinh \psi \sin \theta \cos \phi, \\
Z_{3}=a \sinh (\tau / a) \sinh \psi \sin \theta \sin \phi,
\end{gathered}
$$

and the de Sitter line element takes the form

$$
\begin{aligned}
d s^{2}= & d \tau^{2}-a^{2} \sinh ^{2}(\tau / a) \\
& \times\left[d \psi^{2}+\sinh ^{2} \psi\left(d \theta^{2}+\sin ^{2} \theta d \phi^{2}\right)\right] .
\end{aligned}
$$

However, it may be noted that these coordinates only cover part of the hyperboloid.

Again it is convenient to introduce a conformal time defined here by

$$
\eta_{n}= \begin{cases}2 \operatorname{arccoth} e^{\tau / a} & \text { for } \tau \geqslant 0 \\ 2 \operatorname{arctanh} e^{\tau / a} & \text { for } \tau \leqslant 0\end{cases}
$$

It may be noted that, as $\tau$ increases from $-\infty$ to 0 and then to $\infty, \eta_{n}$ increases from 0 to $\infty$ and then decreases back to 0 . Using this, the hyperboloid is parametrized by Eqs. (4.6) where $\sinh (\tau / a)= \pm 1 / \sinh \eta_{n}, \quad \cosh (\tau / a)=\cosh \eta_{n} / \sinh \eta_{n}$, and the line element (4.7) becomes

$$
d s^{2}=\frac{a^{2}}{\sinh ^{2} \eta_{n}}\left[d \eta_{n}^{2}-d \psi^{2}-\sinh ^{2} \psi\left(d \theta^{2}+\sin ^{2} \theta d \phi^{2}\right)\right] .
$$

Since

$$
Z_{0}+Z_{1}=\frac{a}{\sinh \eta_{n}}\left(\cosh \eta_{n} \pm \cosh \psi\right)
$$

we have

$$
\delta\left(Z_{0}+Z_{1}\right)=\frac{1}{a}\left[\delta\left(\eta_{n}-\psi\right)+\delta\left(\eta_{n}+\psi\right)\right] .
$$

Thus, on the impulsive wave, $\psi= \pm \eta_{n}$ and it can again be shown that the impulsive addition to the de Sitter line element (4.9) is given by

$$
\begin{aligned}
d s_{1}^{2}= & 4 p a\left[2-\cos \theta \ln \left|\frac{1+\cos \theta}{1-\cos \theta}\right|\right] \\
& \times\left[\delta\left(\eta_{n}-\psi\right)\left(d \eta_{n}-d \psi\right)^{2}+\delta\left(\eta_{n}+\psi\right)\left(d \eta_{n}+d \psi\right)^{2}\right] .
\end{aligned}
$$

This again looks like two impulses. However, the first term is nonvanishing only for $\psi>0$, while the second term is nonvanishing only for $\psi<0$. Both components are required for the conformal picture to be geodesically complete. This is described in Fig. 6.

The spherical pulse is now propagating in a three-space $\tau=$ const which has a negative curvature and contracts for $\tau<0$ according to $a|\sinh (\tau / a)|$. The impulse is located at $|\psi|=\eta_{n}=2 \operatorname{arctanh} e^{\tau / a}$; i.e., it is expanding from $\psi=0$ to $|\psi|=\infty$.

\section{NULL PARTICLE IN AN ANTI-de SITTER BACKGROUND}

For a vacuum universe with a negative cosmological constant $(\Lambda<0)$, the Schwarzschild-anti-de Sitter solution can be written in the form (2.1) but with $a^{2}$ replaced by $-a^{2}$. It is then possible to proceed as in Sec. II to boost the singular 


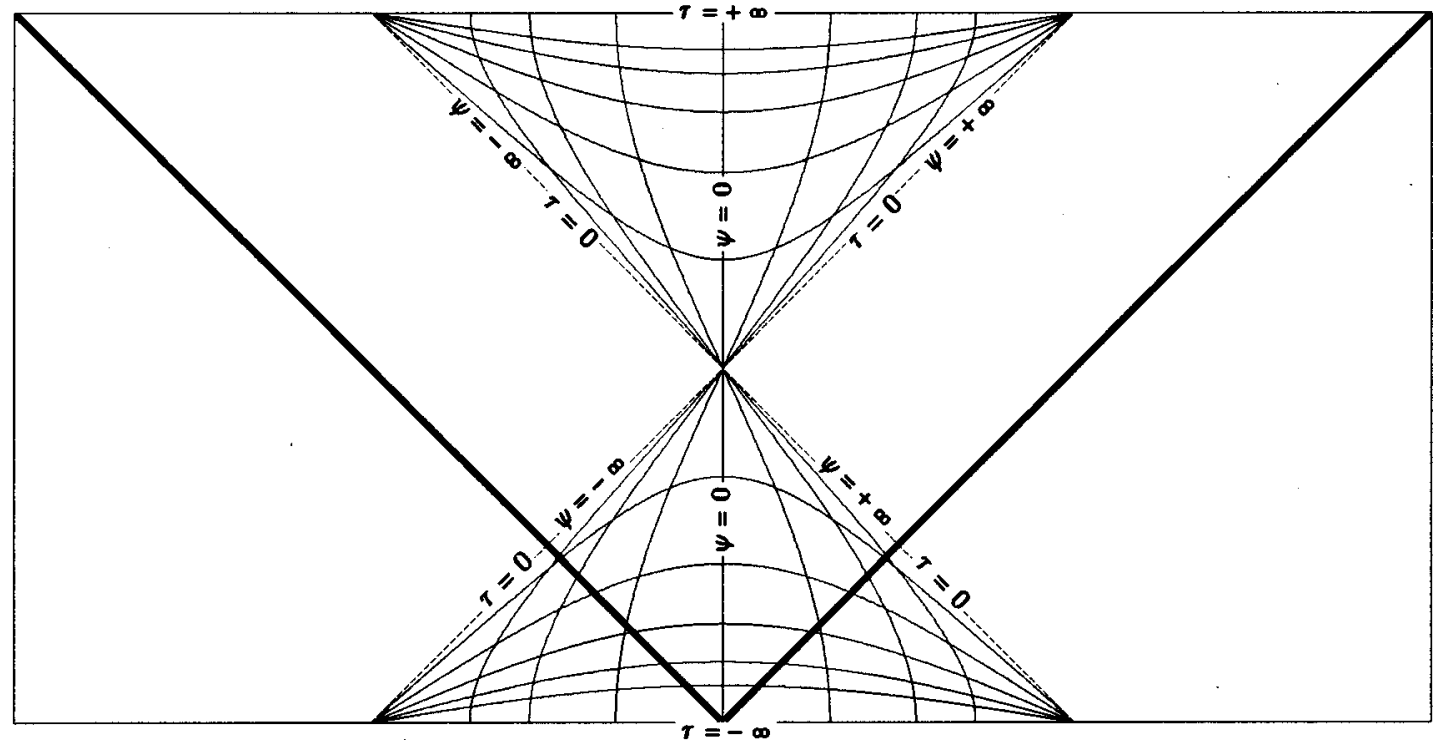

FIG. 6. The conformal diagram of the de Sitter space-time with coordinates $\tau, \psi$. For the corresponding comoving observers the spherical impulsive wave expands in a contracting space of negative curvature.

point $r=0$ in the limit as $m \rightarrow 0$ in an appropriate way. This again produces an impulsive wave, but this time in an anti-de Sitter background.

Before proceeding, however, it is first appropriate to recall that the anti-de Sitter solution can be represented as the four-dimensional hyperboloid

$$
Z_{0}^{2}-Z_{1}^{2}-Z_{2}^{2}-Z_{3}^{2}+Z_{4}^{2}=a^{2}
$$

embedded in a five-dimensional Minkowski space-time

$$
d s^{2}=d Z_{0}^{2}-d Z_{1}^{2}-d Z_{2}^{2}-d Z_{3}^{2}+d Z_{4}^{2}
$$

with two timelike dimensions $Z_{0}$ and $Z_{4}$. The natural parametrization of this is given by

$$
\begin{gathered}
Z_{4}=\sqrt{a^{2}+r^{2}} \cos (t / a), \\
Z_{0}=\sqrt{a^{2}+r^{2}} \sin (t / a), \\
Z_{1}=r \cos \theta, \\
Z_{2}=r \sin \theta \cos \phi, \\
Z_{3}=r \sin \theta \sin \phi .
\end{gathered}
$$

From this it can be seen that a source at the origin $r=0$ is located on the line

$$
\left(Z_{0}, Z_{1}, Z_{2}, Z_{3}, Z_{4}\right)=(a \sin (t / a), 0,0,0, a \cos (t / a)),
$$

which is a closed timelike geodesic which returns to the same event after $\Delta t=2 \pi a$. This is illustrated in Fig. 7 in which the spacelike coordinates $Z_{2}$ and $Z_{3}$ are suppressed. It can thus be seen that the $m \rightarrow 0$ limit of the Schwarzschildanti-de Sitter solution has a single source following a closed timelike line.

In this case, the first order perturbation of the anti-de Sitter line element, equivalent to Eq. (2.7), can be written as

$$
\begin{aligned}
d s_{1}^{2}= & -\frac{2 m a^{2}}{r}\left[\left(\frac{Z_{4} d Z_{0}-Z_{0} d Z_{4}}{Z_{4}^{2}+Z_{0}^{2}}\right)^{2}\right. \\
& \left.+\frac{a^{2}}{r^{2}}\left(\frac{Z_{0} d Z_{0}+Z_{4} d Z_{4}}{Z_{4}^{2}+Z_{0}^{2}}\right)^{2}\right]
\end{aligned}
$$

where $r=\left(Z_{0}^{2}+Z_{4}^{2}-a^{2}\right)^{1 / 2}$. A boost can then be performed in the $Z_{1}$ direction using Eqs. (2.8). Again it can be seen that, in the limit as $v \rightarrow 1$, this linear perturbation term vanishes

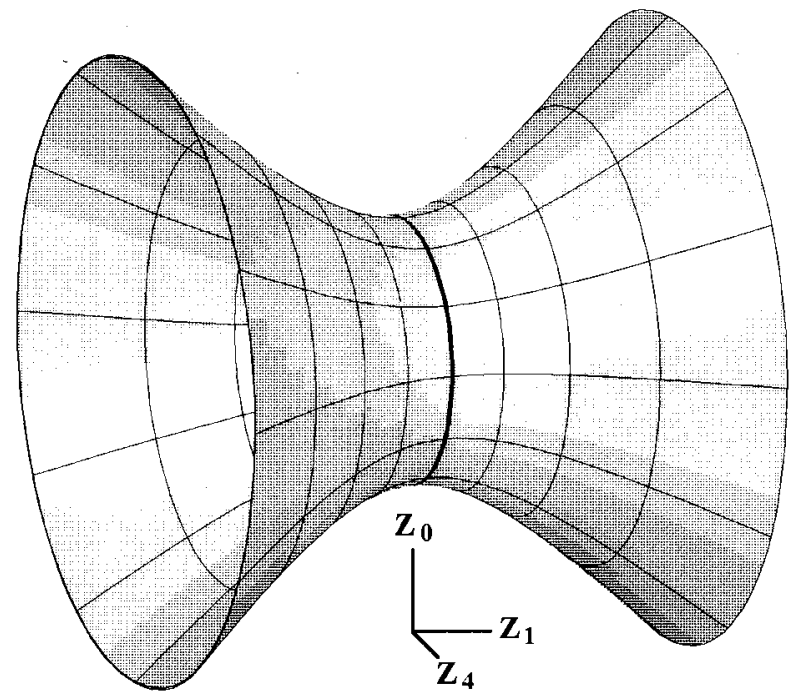

FIG. 7. The anti-de Sitter space-time represented as a fourdimensional hyperboloid embedded in a five-dimensional spacetime with two timelike dimensions $Z_{0}$ and $Z_{4}$. Here the coordinates $Z_{2}$ and $Z_{3}$ have been suppressed, and the closed world line of the particle which corresponds to the weak limit of the source of the Schwarzschild-anti-de Sitter space-time is indicated. 


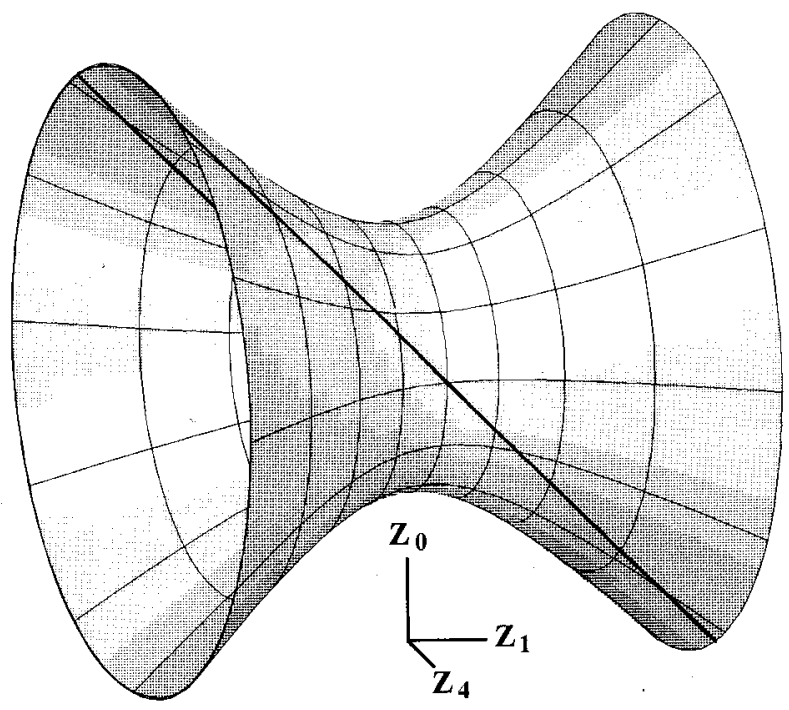

FIG. 8. The anti-de Sitter space-time represented as a fourdimensional hyperboloid embedded in a five-dimensional Minkowski space-time with two timelike dimensions $Z_{0}$ and $Z_{4}$. The null world line is indicated by the particle which correspond to the source of the Schwarzschild-anti-de Sitter space-time boosted to the limit in which its velocity is that of light and its mass vanishes.

everywhere except on the hypersurface $Z_{0}+Z_{1}=0$. By putting $m=p \sqrt{1-v^{2}}$, the line element then takes the form

$$
\begin{aligned}
d s^{2}= & d Z_{0}^{2}-d Z_{1}^{2}-d Z_{2}^{2}-d Z_{3}^{2}+d Z_{4}^{2} \\
& -2 p a^{2} H\left(Z_{4}\right) \delta\left(Z_{0}+Z_{1}\right)\left(d Z_{0}+d Z_{1}\right)^{2},
\end{aligned}
$$

where using Eq. (2.11) with $x^{2}=\left(Z_{0}+v Z_{1}\right)^{2} /\left(1-v^{2}\right)$,

$$
H\left(Z_{4}\right)=\int_{-\infty}^{\infty} \frac{\left(Z_{4}^{2}-a^{2}\right) Z_{4}^{2}+\left(a^{2}+Z_{4}^{2}\right) x^{2}}{\left(Z_{4}^{2}-a^{2}+x^{2}\right)^{3 / 2}\left(Z_{4}^{2}+x^{2}\right)^{2}} d x .
$$

Since in this case the integrand is nonsingular, we obtain, immediately,

$$
H\left(Z_{4}\right)=-\frac{2}{a^{2}}\left[2-\frac{Z_{4}}{a} \ln \left|\frac{a+Z_{4}}{a-Z_{4}}\right|\right] .
$$

It can be seen that in this case the resulting space-time differs from the anti-de Sitter space-time by the inclusion of an impulsive wave located on the null hypersurface given by

$$
Z_{0}+Z_{1}=0, \quad Z_{4}^{2}-Z_{2}^{2}-Z_{3}^{2}=a^{2} .
$$

Unlike the case in a de Sitter background, this is now an evolving two-hyperboloid in a five-dimensional Minkowski space with two time dimensions. line

Also, the source is now a null particle having the world

$$
Z_{0}+Z_{1}=0, \quad Z_{2}=Z_{3}=0, \quad Z_{4}= \pm a .
$$

This is represented by the two null straight lines on the fourdimensional hyperboloid as indicated in Fig. 8. However, since time is here effectively wrapped around the hyperbo- loid, this represents a null particle which propagates to infinity and then comes back again in an endless cycle.

\section{GLOBAL COORDINATE CHART $(\Lambda<0)$}

In an anti-de Sitter space-time, it is appropriate initially to consider the natural global coordinate system obtained by putting $r=a \sinh R$ in Eqs. (5.3) by which

$$
\begin{gathered}
Z_{4}=a \cosh R \cos (t / a), \\
Z_{0}=a \cosh R \sin (t / a), \\
Z_{1}=a \sinh R \cos \theta, \\
Z_{2}=a \sinh R \sin \theta \cos \phi, \\
Z_{3}=a \sinh R \sin \theta \sin \phi,
\end{gathered}
$$

and the line element becomes

$$
d s^{2}=\cosh ^{2} R d t^{2}-a^{2}\left[d R^{2}+\sinh ^{2} R\left(d \theta^{2}+\sin ^{2} \theta d \phi^{2}\right)\right] .
$$

In this case, the privileged family of timelike observers have world lines on which $R, \theta$, and $\phi$ are constant, and their proper times are given by $\tau=t \cosh R$. The timelike slices $t=$ const are three-dimensional spaces of constant negative curvature.

We may now consider the impulsive wave on which $Z_{0}+Z_{1}=0$. It can be seen that this is given by

$$
\tanh R \cos \theta=-\sin (t / a) .
$$

It may be noticed that this does not give such a convenient coordinate condition as does the equivalent case in a de Sitter background. Nevertheless, we can show that

$$
\delta\left(Z_{0}+Z_{1}\right)=\frac{\delta\left(t+a \arcsin ^{-1}(\tanh R \cos \theta)\right)}{\sqrt{1+\sinh ^{2} R \sin ^{2} \theta}},
$$

and, evaluating $H\left(Z_{4}\right)$ using Eqs. (6.1), we obtain that the impulsive addition to the anti-de Sitter line element (6.2) is given by

$$
\begin{aligned}
d s_{1}^{2}= & 4 p\left[\frac{2}{\cosh R \cos (t / a)}-\ln \left|\frac{1+\cosh R \cos (t / a)}{1-\cosh R \cos (t / a)}\right|\right] \\
& \times \delta(t+a \arcsin (\tanh R \cos \theta))\left(a \frac{\cos \theta}{\cosh R} d R\right. \\
& -a \sinh R \sin \theta d \theta+\cosh R \cos (t / a) d t)^{2}
\end{aligned}
$$

which looks somewhat complicated.

We would now like to visualize this wave as it would be observed by the natural family of observers in the threedimensional space $(R, \theta, \phi)$. For this, we consider a section $t=$ const through the space-time. It is then convenient to introduce the coordinate $\widetilde{Z}_{0}=a \cosh R$ which, together with the coordinates $Z_{1}, Z_{2}$, and $Z_{3}$ span a four-dimensional pseudo-Euclidean space 


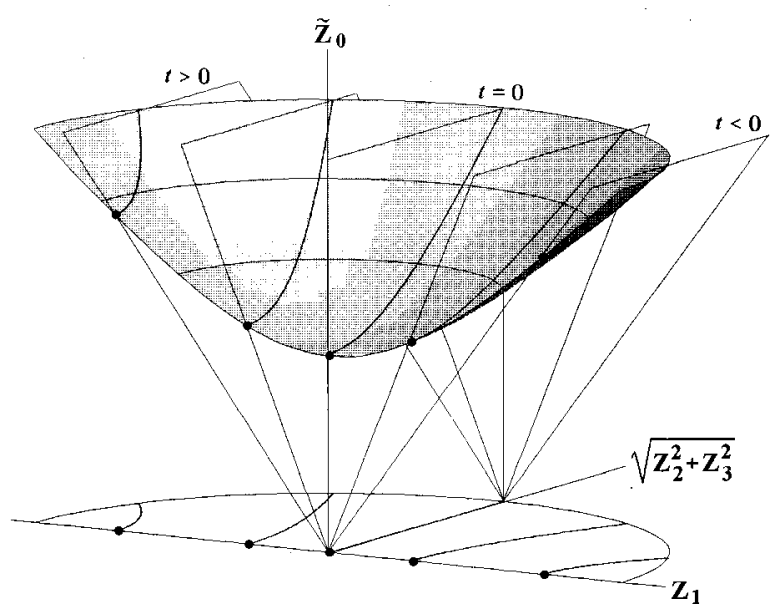

FIG. 9. The geometry of the impulsive wave in anti-de Sitter space-time as observed by natural family of observers at any time is given by the intersection of the plane $Z_{1}=-\sin (t / a) \widetilde{Z}_{0}$ with half of a rotational hyperboloid in the pseudo-Euclidean space so that the wavefronts are the spaces of constant negative curvature.

$$
d s_{4}^{2}=d \widetilde{Z}_{0}^{2}-d Z_{1}^{2}-d Z_{2}^{2}-d Z_{3}^{2}
$$

The hypersurface of the impulsive wave can now be visualized at any time as the intersection of the plane $Z_{1}=-\sin (t / a) \widetilde{Z}_{0}$ with half of a rotational hyperboloid

$$
\widetilde{Z_{0}^{2}}-Z_{1}^{2}-\left(\sqrt{Z_{2}^{2}+Z_{3}^{2}}\right)^{2}=a^{2}
$$

in the pseudo-Euclidean half space $\left(\widetilde{Z}_{0}, Z_{1}, \sqrt{Z_{2}^{2}+Z_{3}^{2}}\right)$ representing the three-dimensional space of constant negative curvature with the metric $d s_{3}^{2}=-a^{2}\left[d R^{2}+\sinh ^{2} R\left(d \theta^{2}\right.\right.$ $\left.\left.+\sin ^{2} \theta d \phi^{2}\right)\right]$. This is illustrated in Fig. 9.

The source of the impulsive wave is a single null particle located on the wave at the point $\sqrt{Z_{2}^{2}+Z_{3}^{2}}=0$, $Z_{1}=-a \tan (t / a)$. This indicates that the null particle moves from $Z_{1}=\infty$ to $Z_{1}=-\infty$ as $t$ goes from $(-\pi / 2) a$ to $(\pi / 2) a$.

It is then possible to visualize the wavefront at any time by projecting the intersection of the plane and the hyperboloid onto the space of $Z_{1}$ and $\sqrt{Z_{2}^{2}+Z_{3}^{2}}$ as illustrated in Fig. 9 , and then rotating it about the $Z_{1}$ axis. In this way, the $Z_{2}$ and $Z_{3}$ coordinates are reintroduced by putting

$$
Z_{2}=\sqrt{Z_{2}^{2}+Z_{3}^{2}} \cos \phi, \quad Z_{3}=\sqrt{Z_{2}^{2}+Z_{3}^{2}} \sin \phi .
$$

The impulsive wave is thus represented by the sequence of hyperboloidal surfaces illustrated in Fig. 10. While the angle $\phi$ is a "polar" angle associated with the axial symmetry of the surfaces, the angle $\theta$ is measured from the (positiveoriented) axis of symmetry. It can be shown that each hyperboloidal surface representing the impulsive wave front has an asymptotic angle $\theta_{a}$ given by $\theta_{a}=t / a+\pi / 2$. Therefore, as $t / a$ grows from $-\pi / 2$ to $\pi / 2$ (and the source null particle moves from $Z_{1}=\infty$ to $\left.Z_{1}=-\infty\right)$ the wave surfaces open from $\theta_{a}=0$ to $\theta_{a}=\pi$.

Since the time coordinate $t$ is periodic, it can be seen that the impulsive wave propagates to $Z_{1}=-\infty$ and then back to $Z_{1}=\infty$ in an endless cycle.

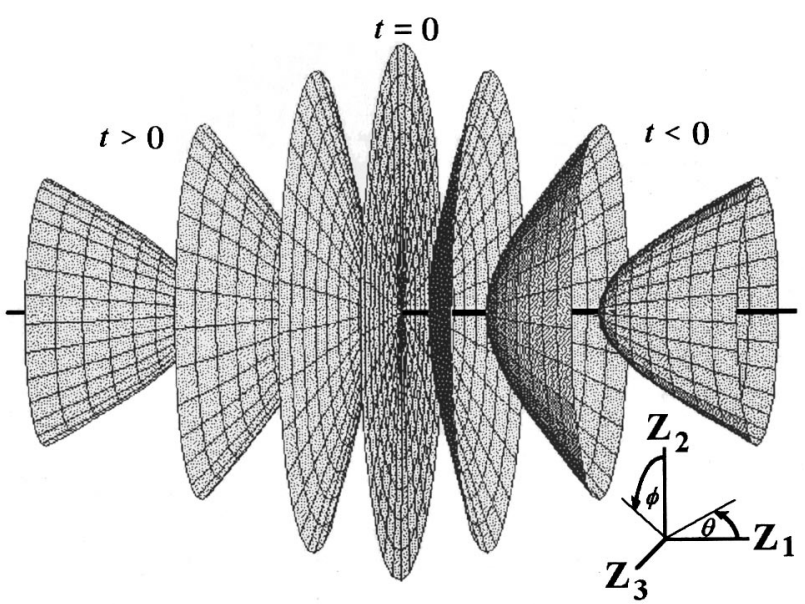

FIG. 10. By projecting the intersections representing the wave fronts from Fig. 9 onto the plane of $Z_{1}, Z_{2}^{2}+Z_{3}^{2}$ and rotating them about the $Z_{1}$ axis we get the visualization of the impulsive wave in the anti-de Sitter space-time as a sequence of hyperboloidal surfaces. The source of the wave is a null particle moving along the axis of symmetry which at any time is located in the corresponding surface.

In order to investigate the conformal structure of the space-time in these coordinates, we introduce the conformal time $\chi$ defined by

$$
\chi=2 \arctan \left(e^{R}\right)-\pi / 2 .
$$

In this case we have $\cosh R=\sec \chi, \sinh R=\tan \chi$, and the line element becomes

$$
d s^{2}=\frac{a^{2}}{\cos ^{2} \chi}\left(\frac{1}{a^{2}} d t^{2}-d \chi^{2}-\sin ^{2} \chi\left(d \theta^{2}+\sin ^{2} \theta d \phi^{2}\right)\right) .
$$

In this case, the impulsive wave on which $Z_{0}+Z_{1}=0$ can be seen to occur when $\sin \chi \cos \theta=-\sin (t / a)$ in agreement with Eq. (6.3). The location of the wave in the conformal diagram of the anti-de Sitter space-time for $\theta=0$ is shown in Fig. 11.

\section{OTHER COORDINATE CHARTS $(\Lambda<0)$}

Let us now consider how the impulsive wave would appear to the alternative family of observers which, at any synchronous time, are located in a space of constant negative curvature. In this case, the four-dimensional hyperboloid (5.1) can be parametrized by

$$
\begin{gathered}
Z_{0}=a \sin (\tau / a), \\
Z_{1}=a \cos (\tau / a) \sinh \psi \cos \theta, \\
Z_{2}=a \cos (\tau / a) \sinh \psi \sin \theta \cos \phi, \\
Z_{3}=a \cos (\tau / a) \sinh \psi \sin \theta \sin \phi, \\
Z_{4}=a \cos (\tau / a) \cosh \psi,
\end{gathered}
$$

and the anti-de Sitter line element takes the form 


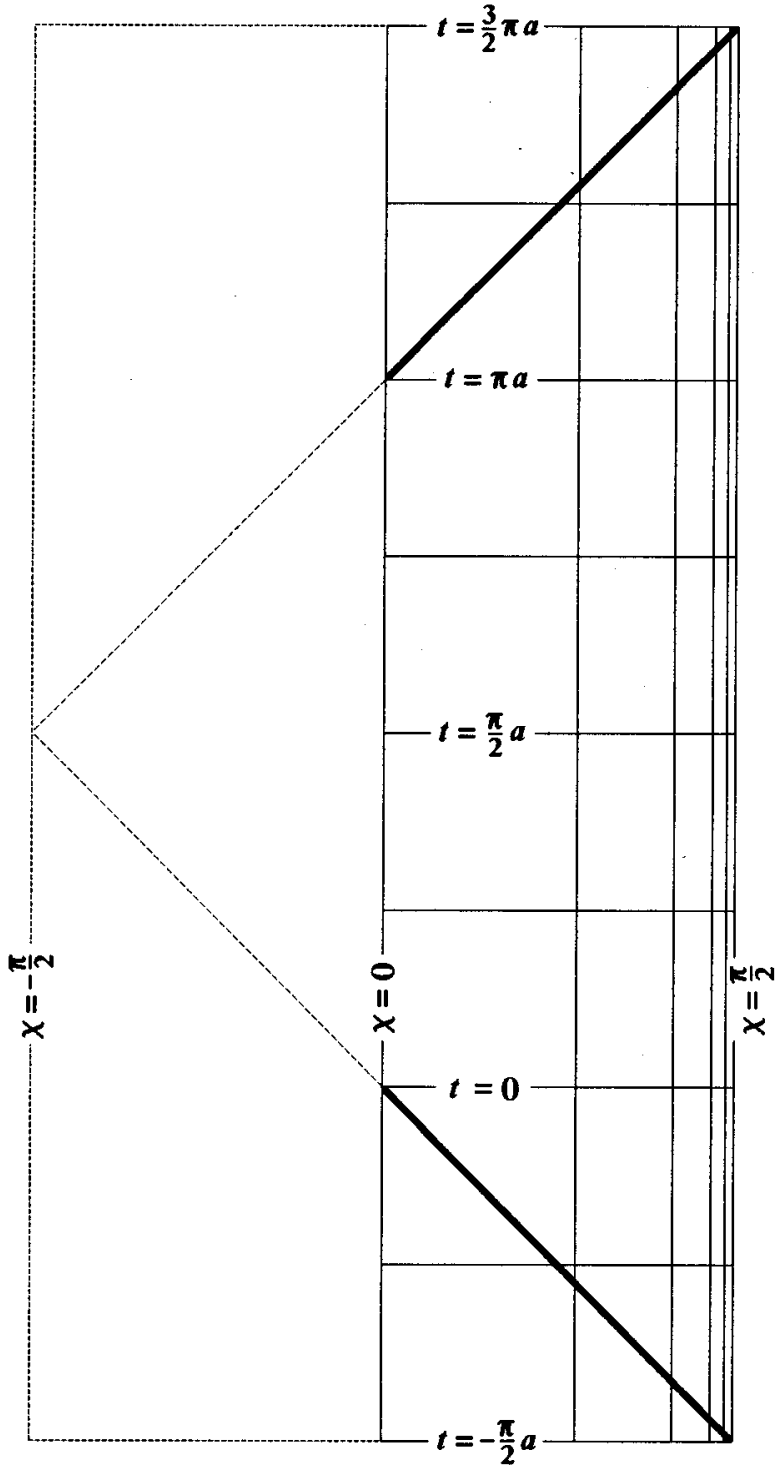

FIG. 11. The conformal diagram of the anti-de Sitter spacetime with natural global coordinate chart $t, \chi$. Each point represents a two-sphere parametrized by $\theta, \phi$, antipodal points on the spheres correspond to $\pm \chi$. Null and spacelike infinity form a timelike surface $\chi=\pi / 2(R=\infty)$. The impulsive wave for $\theta=0$ is also indicated.

$d s^{2}=d \tau^{2}-a^{2} \cos ^{2}(\tau / a)\left[d \psi^{2}+\sinh ^{2} \psi\left(d \theta^{2}+\sin ^{2} \theta d \phi^{2}\right)\right]$.

The time-slices $\tau=$ const are three-dimensional spaces of constant negative curvature, but which do not have a constant "size" as occurs for the global coordinates (6.2) in the previous section. Their curvatures change according to $a \cos (\tau / a)$. However, it may be noted that these coordinates only cover part of the hyperboloid.

Again it is convenient to introduce a conformal time defined by

$$
\eta_{n}=\operatorname{arctanh}[\sin (\tau / a)]
$$

It may be noted that, as $\tau / a$ increases from $-\pi / 2$ to 0 and then to $\pi / 2, \eta_{n}$ increases from $-\infty$ to $\infty$. With these param-

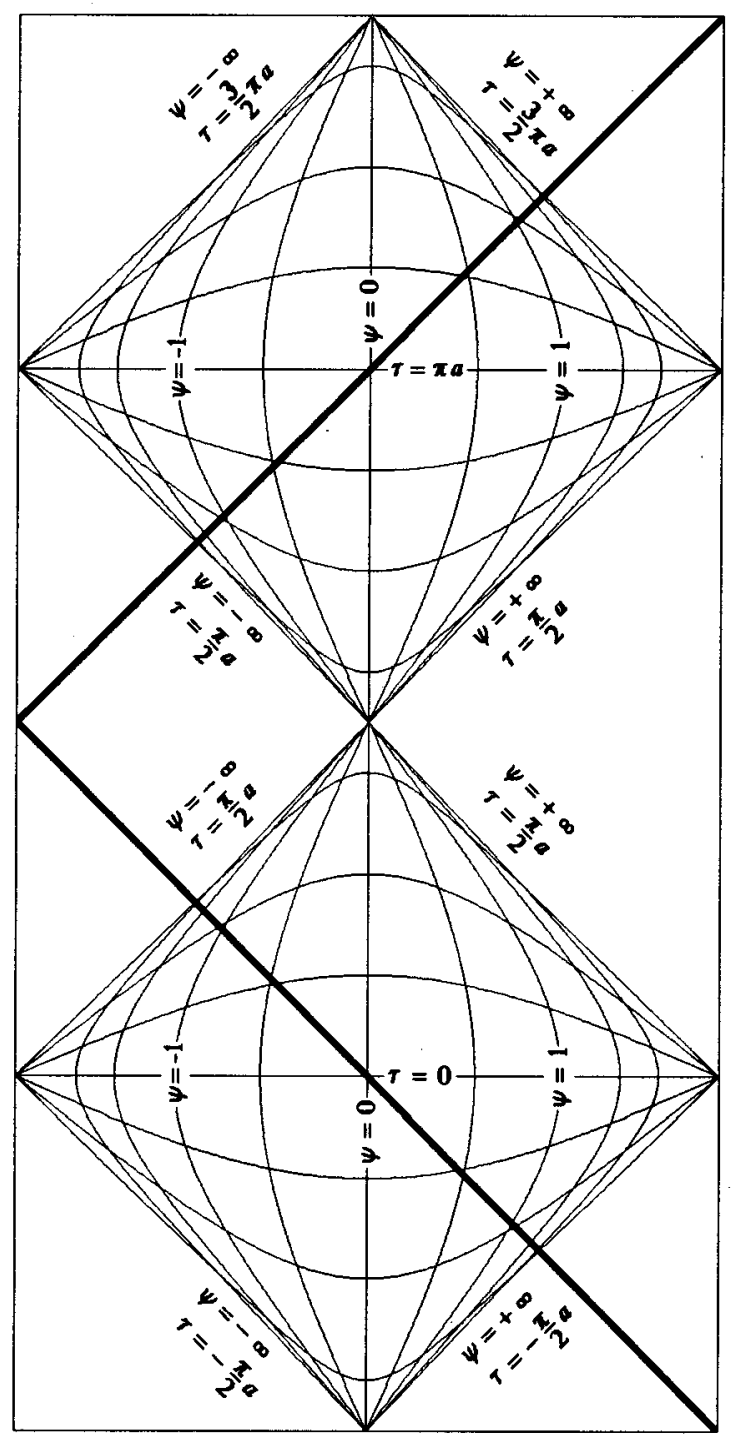

FIG. 12 . The synchronous coordinates $\tau, \psi, \theta, \phi$ cover only a part of the whole anti-de Sitter space-time. The timelike geodesics $\psi, \theta, \phi$ constants all converge. In this diagram drawn for $\theta=0$ the impulsive wave propagates along the indicated null lines.

eters, the hyperboloid is now given by Eqs. (7.1) with $\sin (\tau / a)=\tanh \eta_{n}, \cos (\tau / a)=\operatorname{sech} \eta_{n}$, and line element (7.2) becomes

$$
d s^{2}=\frac{a^{2}}{\cosh ^{2} \eta_{n}}\left[d \eta_{n}^{2}-d \psi^{2}-\sinh ^{2} \psi\left(d \theta^{2}+\sin ^{2} \theta d \phi^{2}\right)\right]
$$

Proceeding as before, we can consider the impulsive wave on which $Z_{0}+Z_{1}=0$. However, this again does not give such a convenient coordinate condition as does the equivalent case in a de Sitter background. Calculations can again be performed to yield an expression similar to Eq. (6.4). However, this does not provide any further insights. The conformal diagram with the impulse for $\theta=0$ is given in Fig. 12.

It is finally appropriate here to consider a conformally flat coordinate system. Accordingly, we can put 


$$
\begin{gathered}
Z_{0}=\frac{1}{2 x}\left[a^{2}-\eta^{2}+x^{2}+y^{2}+(z-a)^{2}\right], \\
Z_{1}=\frac{a}{x}(z-a), \\
Z_{2}=\frac{1}{2 x}\left[a^{2}+\eta^{2}-x^{2}-y^{2}-(z-a)^{2}\right], \\
Z_{3}=\frac{a}{x} y, \\
Z_{4}=\frac{a}{x} \eta .
\end{gathered}
$$

With this, the line element becomes

$$
d s^{2}=\frac{a^{2}}{x^{2}}\left(d \eta^{2}-d x^{2}-d y^{2}-d z^{2}\right)
$$

It is also convenient to put

$$
x=\rho \cos \theta, \quad y=\rho \sin \theta \cos \phi, \quad z=\rho \sin \theta \sin \phi,
$$

so that the line element becomes

$$
d s^{2}=\frac{a^{2}}{\rho^{2} \cos ^{2} \theta}\left[d \eta^{2}-d \rho^{2}-\rho^{2}\left(d \theta^{2}+\sin ^{2} \theta d \phi^{2}\right)\right] .
$$

It can also be shown that the form of the impulse is given by

$$
\delta\left(Z_{0}+Z_{1}\right)=\frac{|x|}{\rho}[\delta(\eta-\rho)+\delta(\eta+\rho)]
$$

and the impulsive addition to the anti-de Sitter line element is given by

$$
\begin{aligned}
d s_{1}^{2}= & \frac{4 p}{|\cos \theta|}\left[2-\frac{1}{\cos \theta} \ln \left|\frac{1+\cos \theta}{1-\cos \theta}\right|\right] \\
& \times\left[\delta(\eta-\rho)(d \eta-d \rho)^{2}+\delta(\eta+\rho)(d \eta+d \rho)^{2}\right] .
\end{aligned}
$$

This is clearly the simplest coordinate form of the shock geometry (5.6) in an anti-de Sitter background. Again this is only one impulse for $\eta>0$ and $\eta<0$. Also, the impulsive wave is given by $\eta= \pm \rho$. The metric is regular for $\theta=\pi / 2$ so that it is singular only for $\theta=0$ and $\theta=\pi$. The conformal diagram is given in Fig. 13.

For any value of $\eta$, the surfaces $\rho=$ const according to Eqs. (7.7) look like spheres. However, they are not spheres: They are surfaces that are conformal to spheres. Actually, they are hyperboloidal. Indeed, performing the transformation $\xi=\operatorname{arctanh}(\sin \theta)$ we can write the section $\eta= \pm \rho$ of the line element (7.8) as $d \sigma^{2}=-a^{2}\left(d \xi^{2}+\sinh ^{2} \xi d \phi^{2}\right)$. This represents the hyperboloidal two-space of constant negative curvature $-a$.

We may thus conclude that the conformally flat coordinate system (7.8) is well adapted for describing the impul-

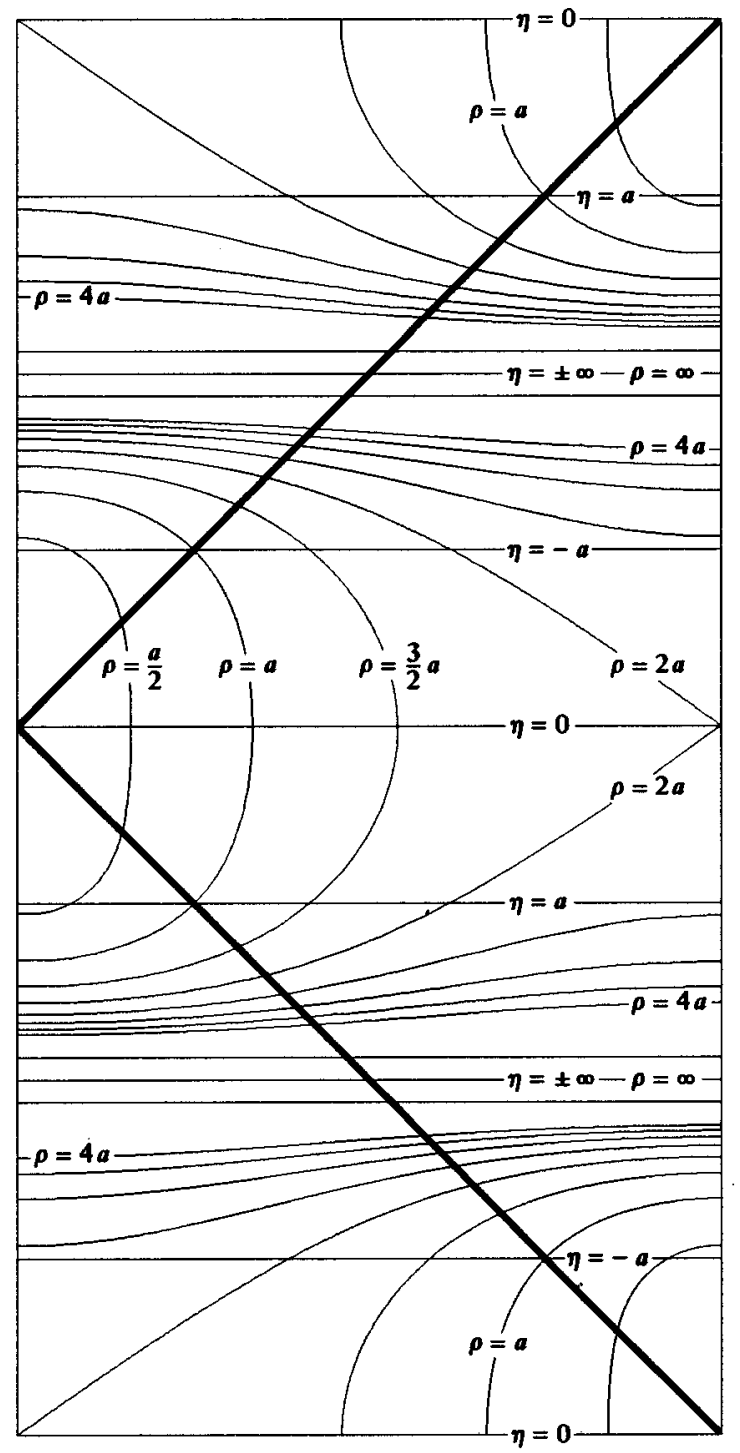

FIG. 13. The conformal diagram of the anti-de Sitter spacetime and conformally flat coordinates $\eta, \rho$ which cover the whole universe. Here we consider the section $Z_{2}=Z_{3}=0$ so that $\phi=\pi / 2$ and $\sin \theta=\left(\rho^{2}-\eta^{2}\right) /(2 a \rho)$. The impulsive wave is given simply by $\eta= \pm \rho$.

sive wave (5.6) in anti-de Sitter space-time. Conversely, the coordinates (6.2) and (7.2) are not suitable due to the fact that their corresponding two-surfaces are spheres having the line element $d \sigma^{2}=-C^{2}\left(d \theta^{2}+\sin ^{2} \theta d \phi^{2}\right), C=$ const. This naturally explains the complicated forms of the impulsive additions to the anti-de Sitter line element (6.4) in these coordinates. By contrast, we have previously observed that all three coordinate systems (3.2), (4.2), and (4.7) that we have introduced in the $\Lambda>0$ case are suitable for describing spherical impulsive gravitational waves in the de Sitter universe since they also have $d \sigma^{2}=-C^{2}\left(d \theta^{2}+\sin ^{2} \theta d \phi^{2}\right)$.

\section{CONCLUSIONS}

Following the method of Hotta and Tanaka [6], we have boosted the Schwarzschild-de Sitter and the Schwarzschildanti-de Sitter metrics to the ultrarelatistic limit and have thus constructed exact solutions for impulsive waves in back- 
grounds with a nonzero cosmological constant. In a de Sitter background, a spherical gravitational wave is generated by two null particles propagating in opposite directions. We have used three privileged families of observers in which the constant time surfaces have constant positive, negative, or zero curvature. We have shown that each of these coordinate systems is suitable for describing the geometry of the impulsive wave. In the closed form of the de Sitter universe which contracts to a minimum size and then reexpands, the impulse propagates from the "north pole" to the "south pole." In an expanding flat and open de Sitter universe it is a contracting sphere; alternatively in a contracting universe the sphere is expanding.

By contrast, the impulsive wave generated in an anti-de Sitter background is hyperboloidal. It is generated by a single null particle. We have also shown that it is only the conformally flat coordinates that form a suitable natural parametrization of this solution.

It may also be noted that a family of impulsive spherical gravitational waves has been constructed by Hogan [9], the elements of which appear to propagate either in a de Sitter or an anti-de Sitter background. However, these are not related to the solutions given above.

\section{ACKNOWLEDGMENTS}

J.P. is grateful for hospitality at the Mathematical Sciences Department at Loughborough University and for support from Grant No. GACR-202/96/0206 of the Czech Republic and Grant No. GAUK-230/96 of Charles University while this work was undertaken.
[1] P. C. Aichelburg and R. U. Sexl, Gen. Relativ. Gravit. 2, 303 (1971).

[2] V. Ferrari and P. Pendenza, Gen. Relativ. Gravit. 22, 1105 (1990)

[3] H. Balasin and H. Nachbagauer, Class. Quantum Grav. 12, 707 (1995).

[4] H. Balasin and H. Nachbagauer, Class. Quantum Grav. 13,
731 (1996).

[5] C. O. Loustó and N. Sánchez, Nucl. Phys. B383, 377 (1992).

[6] M. Hotta and M. Tanaka, Class. Quantum Grav. 10, 307 (1993).

[7] J. Bičák and J. Podolský, Phys. Rev. D 52, 887 (1995).

[8] E. Eriksen and Ф. Grøn, Int. J. Mod. Phys. D 4, 115 (1995).

[9] P. A. Hogan, Phys. Lett. A 171, 21 (1992). 